\title{
Design of the Acoustic Signal Receiving Unit of Acoustic Telemetry While Drilling
}

\author{
Li Zhigang, Ge Shitong, Fu Zhongyao \\ School of Petroleum Engineering, China University of Petroleum (East China), Qingdao, Shandong, China, 266580
}

\begin{abstract}
Signal receiving unit is one of the core units of the acoustic telemetry system. A new type of acoustic signal receiving unit is designed to solve problems of the existing devices. The unit is a short joint in whole. It not only can receive all the acoustic signals transmitted along the drill string, without losing any signal, but will not bring additional vibration and interference. In addition, the structure of the amplitude transformer is designed, which can amplify the signal amplitude and improve the receiving efficiency. The design of the wireless communication module makes the whole device can be used in normal drilling process when the drill string is rotating. So, it does not interfere with the normal drilling operation.
\end{abstract}

\section{Introduction}

Acoustic Telemetry While Drilling is a new technology for the downhole information acquisition and transmission while drilling. The technology transmits information between surface and downhole using acoustic wave as the carrier and drill string as signal channel. Since entering 21th Century, the technology has made a considerable development. At present, this technology has been commercialized in North America, and has achieved good results, especially in the unbalanced drilling and air drilling [1].

Signal receiving unit is one of the core units of the acoustic telemetry system. Its function is to receive the acoustic wave (signal carrier) along the drill pipe, and then extract the useful information (data) from the carrier to achieve the purpose of signal transmission.

The acoustic signal receiving device which is currently in use is generally used in the form of a clamp [2]. These devices use a clamping structure fastening the sound wave receiving sensor and related equipment on the Kelly or the lower drill pipe under

Corresponding author;lee18198@126.com the top drive drilling device. They mainly have the following shortcomings.

The measured signal is the vibration and acoustic signals of the clamping structure and drill string, rather than the vibration and acoustic signals of the drill string. Therefore, the received signal must be different from the transmitting signal without the clamping structure. The form of the clamping structure and the degree of fastening are the main factors that affect the fidelity and reliability of the signal.

As a result of this measurement method, which was completed by an external clamping structure, some high frequency and weak acoustic signal will be lost or filtered.

Because the clamping structure is not a part of the drill string, it is a subsidiary structure, so it will come with the additional vibration and noise, which can interfere with the measurement and reception of the acoustic signal.

\section{Overall structure}

For shortcomings of the existing equipment, this paper designs a new type of acoustic signal receiving 
unit, as shown in Figure 1. The unit is composed of three parts, the external protection sleeve 2, the acoustic signal receiving module 4 and the lower joint 12 , and the three is connected with the screw thread. The lower joint is connected with the external protective sleeve through the screw thread 10. The acoustic signal receiving module connects the lower joint by the screw thread 8 . The unit is a short joint in whole, when used in connection in the drill string, and is a part of the drill string. The whole unit is connected with the other lower part by the external screw thread 13, and the upper part by means of an internal thread 1 .

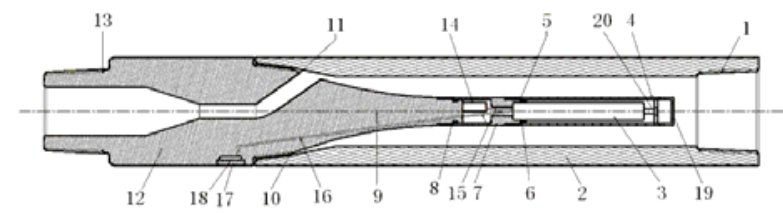

Fig.1 The overall structure of the acoustic signal receiving

device

1-internal thread,2- external protection sleeve,3-circuit board,4- acoustic signal receiving module,5-screw thread,6-signal processing module,7-transducer sleeve,8screw thread,9-amplitude transformer,10- screw thread,11-hole,12-lower joint,13-external screw thread,14-signal receiving transducer,15-cable,16-slender hole,17-cover,18-slot,19-cap,20-communication interface.

The part above the thread 10 of the lower joint is made to be an amplitude transformer structure. There are three holes along the circumferential direction (120 degrees apart) in it. On the outer wall of the lower joint, a slot 18 is provided for placing the wireless communication module. The slot was covered by the nonmetallic cover 17 . The acoustic wave receiving transducer 14 is installed on the upper end face of the amplitude transformer. There is a slender hole 16 going through the lower joint between the upper end face of the amplitude transformer 9 and the slot 18. Electrical cable can be put into the hole and connect the wireless communication module and the acoustic wave signal receiving module.
The acoustic signal receiving module 4 is composed of the sensor sleeve 7 and the signal processing module 6 . The sensor sleeve and the signal processing module are connected through the screw 5. There is a bracket in the sleeve of the signal processing module. The acoustic signal receiving and process circuit 3 can be mounted on it. The circuit is connected with the sensor and the wireless communication module by electric cable 15 . In the signal processing module, the upper part of the sleeve 6 is provided with a communication interface 20, which can be connected with other upper equipment through the interface. The upper part of the sleeve 6 is provided with a thread. When there is other top structure, the sleeve can be connected through the thread; no other equipment, capped and sealed by cover 19 .

Principle: With the tight connection of the lower joint and the lower drill string by the screw thread, the receiving unit can transfer the acoustic wave coming from the lower string while connect the drill string and itself. The sound receiving sensor mounted on the amplitude transformer structure receives the acoustic signal and converts it into an electrical signal. The signal is received and processed by the signal receiving and processing module. The processed signal and data can be stored in the memory of the acoustic signal receiving module, also can be transmitted to the wireless communication module in the outer wall of the lower joint by the cable placed in the inner hole of the lower joint. Furthermore, the wireless communication module transmits the signal to other external communication equipment or surface signal receiving and processing equipment. Due to the existence of the wireless communication module, the signal can be transmitted through the wireless way to other devices on the rig floor or ground, rather than to receive signals through a cable connection. Therefore, the unit can be used in normal drilling or while drilling, in which the drill string is rotating, and it does not interfere with the normal drilling operation. 


\section{The structure of amplitude transformer}

In order to improve the receiving efficiency of the acoustic wave signal, the amplitude transformer structure is used in the front end of the acoustic signal receiving sensor. The amplitude transformer structure can condense the sonic energy from the larger back-end area to the smaller front-end surface where the sensor is mounted. Assuming the area of the back section is a, the area of the front end section is $b$, the ratio of amplitude transforming will be $\lambda=a / b$ $(\lambda>1)$ [3]. The sonic energy is magnifying in $\lambda$ times by the amplitude transformer structure. The signal also is amplified.

In the unit described here, the size of the front end, connected with the lower drill string, is restricted by the size of the drilling tools. So, the cross-sectional area is fixed. The back end where the sensor will be mounted on is restricted by the size of the sensor and processing circuit, the section area is also fixed, at least lager than the size of the sensor. The works can be done to improve the signal receiving efficiency is to optimize the size and shape of the amplitude transformer structure through researching the relationship between the length of the amplitude transformer and the effect of the amplification. The amplitude transformer was designed as shown in Fig.2.

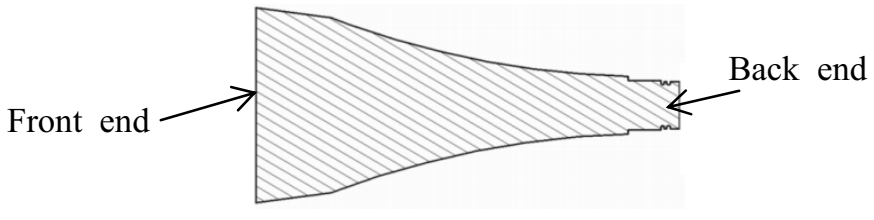

Fig.2 Longitudinal profile of the amplitude transformer

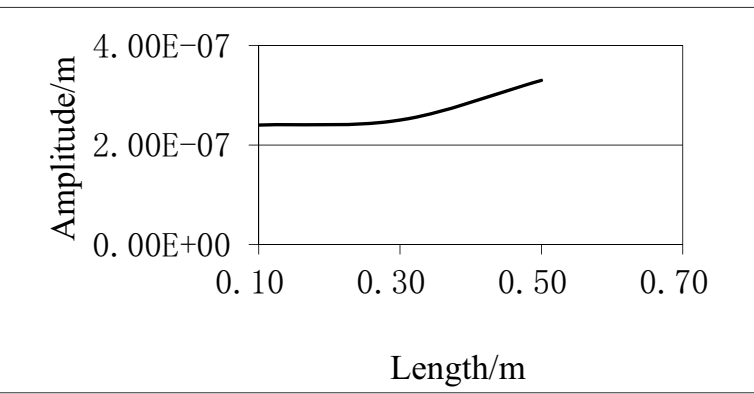

Fig.3 relationship of the length of amplitude transformer and receiving signal amplitude
Through building the three-dimensional solid finite element model, the change of the amplitude of acoustic signal is calculated in the case of different length $(0.1-0.5 \mathrm{~m})$ of the amplitude transformer using finite element method by software ANSYS. In the calculation, a transient excitation signal is applied to the front end of the amplitude transformer, and the vibration amplitude is taken at the middle point of the back end face. The calculated result is shown in Fig.3. As shown in the figure, the amplitude of receiving signal grows up obviously when the length is bigger than $0.3 \mathrm{~m}$ in the unit's sizes. But the length will be too long and influences the unit's strength and performance when the length is larger than $0.5 \mathrm{~m}$.

\section{Conclusion}

To sum up, the unit has the following advantages:

(1)The unit is a short joint, connected to the drill string, can not only completely received all signal transmitted from down hole, but does not produce additional interference, ensure the fidelity and anti-jamming performance. Also, threaded connections are more reliable than clamping form, no prominent structure, does not affect the normal drilling operation.

(2)The acoustic signal receiving module does not participate in the whole structural deformation, so as to ensure the strength. At the same time, it can be disassembled, easy to update and maintain.

(3)The structure of the amplitude transformer is designed to amplify the amplitude of the received signal and improve the receiving efficiency.

(4)The design of the wireless communication module makes the unit can be used while drilling, and does not interfere with the normal drilling operation.

In short, the unit can complete the reception of the acoustic signal efficiently, but also is convenient for the installation, operation and use, does not affect the normal drilling operations, do not increase the 
labor intensity of the drilling site.

\section{Acknowledgements}

The paper is supported by "the Fundamental Research Funds for the Central Universities" 15CX05034A).

\section{References}

1. Reeves Michael, Smith Douglas, and etc. Unique acoustic telemetry network with distributed pressure measurement nodes enables accurate real-time analysis of sweep effectiveness [J]. Proceedings - SPE Annual Technical Conference and Exhibition, v (2), p1690-1709, 2013.

2. Neff J M, Camwell P L. Field-Test Results of an Acoustic MWD System [R]. SPE/IADC 105021. 2007.

3. Lin Zhongmao. Principle and Design of Ultrasonic Amplitude Transformer[M]. Science Press, 1987:55-97. 